\title{
Singular gauge transformations and supersymmetry breakdown on warped orbifolds
}

\author{
Zygmunt Lalak and Radosław Matyszkiewicz \\ Institute of Theoretical Physics \\ University of Warsaw, Poland
}

\begin{abstract}
We have analyzed the breakdown of global supersymmetry by a non-vanishing expectation value of the fifth component of the graviphoton on warped $S^{1} / Z_{2}$ orbifolds. It has been demonstrated that the setups where such a breakdown is possible correspond to the models where the true gauge symmetry on the orbifold, respecting the $Z_{2}$-parities and periodicity, is broken by boundary terms. In the tuned models, giving Randall-Sundrum warp factor, gauge symmetry stays intact, and any $\left\langle A_{5}\right\rangle$ can be gauged away without violating supersymmetry.
\end{abstract}

October 2003 


\section{Introduction}

Brane-bulk supergravities generalize the concept of supersymmetry to setups combining degrees of freedom that propagate in subspaces of various dimensionalities, sometimes spatially disconnected [1]-44. They provide consistent field-theoretic framework for the discussion of important aspects of modern string theory physics. Most importantly, with supergravities that include lower dimensional branes it is possible to study reliably supersymmetry breakdown and its transmission between various sectors of a model. In this note we shall discuss a class of warped (gauged) supergravities with a non-zero background of the fifth component of the graviphoton switched on. It turns out that such a background typically breaks supersymmetry completely leading to four-dimensional $A d S_{4}$ models with no supersymmetric vacua. The exceptional case is that of the models with tuned brane tensions corresponding to the RandallSundrum warped geometry. There the $\left\langle A_{5}\right\rangle$ doesn't break supersymmetry. In the present paper we discuss several examples with various sets of boundary conditions, in particular the ones that correspond to super-bigravities. We also discuss in detail and clarify the relation between the original $U(1)$ gauge invariance that has been instrumental in the construction of the warped supergravities, and the breaking of supersymmetry by non-zero $\left\langle A_{5}\right\rangle$. It turns out that often in order to supersymmetrize the brane-bulk Lagrangian one needs to sacrifice the original gauge invariance. These are precisely the cases where it is possible to break supersymmetry assuming non-vanishing $\left\langle A_{5}\right\rangle$. One can think of such configurations as of the 'would-be' Wilson lines of the broken gauge symmetry. Supersymmetry breakdown due to boundary conditions has been addressed by a number of papers [5]-[13]. In fact, the interesting point is that eventually the origin of supersymmetry violation induced by non-zero $\left\langle A_{5}\right\rangle$ can be traced back to fermionic boundary conditions given by boundary mass terms of gravitini. The boundary masses are supersymmetry singlets, whereas boundary superpotentials, or boundary gaugino condensates, which have been identified earlier as sources of supersymmetry violation, do transform under supersymmetry.

\section{Setup}

To begin with, let us briefly summarize the brane-bulk super-bigravity Lagrangian, constructed in [10]. The simple $\mathrm{N}=2 \mathrm{~d}=5$ supergravity multiplet contains metric tensor (represented by the vielbein $e_{\alpha}^{m}$ ), two gravitini $\Psi_{\alpha}^{A}$ and one vector field $A_{\alpha}$ - the graviphoton. We shall consider gauging of a $U(1)$ subgroup of the global $S U(2)_{R}$ symmetry of the $5 \mathrm{~d}$ bulk Lagrangian. One adds to the initial bulk Lagrangian boundary terms that include brane tensions and/or gravitini mass terms on each brane. The 5 d action describing such a setup reads $S=\int_{M_{5}}\left(\mathcal{L}_{\text {bulk }}+\mathcal{L}_{\text {brane }}\right)$, where

$$
\begin{aligned}
e_{5}^{-1} \mathcal{L}_{\text {bulk }}= & \frac{1}{2} R-\frac{3}{4} \mathcal{F}_{\alpha \beta} \mathcal{F}^{\alpha \beta}-\frac{1}{2 \sqrt{2}} A_{\alpha} \mathcal{F}_{\beta \gamma} \mathcal{F}_{\delta \epsilon} \epsilon^{\alpha \beta \gamma \delta \epsilon} \\
& -\frac{1}{2} \bar{\Psi}_{\alpha}^{A} \gamma^{\alpha \beta \gamma} D_{\beta} \Psi_{\gamma A}+\frac{3 \mathrm{i}}{8 \sqrt{2}}\left(\bar{\Psi}_{\gamma}^{A} \gamma^{\alpha \beta \gamma \delta} \Psi_{\delta A}+2 \bar{\Psi}^{\alpha A} \Psi_{A}^{\beta}\right) \mathcal{F}_{\alpha \beta} \\
& -\frac{\mathrm{i}}{\sqrt{2}} \mathcal{P}_{A B} \bar{\Psi}_{\alpha}^{A} \gamma^{\alpha \beta} \Psi_{\beta}^{B}-\frac{8}{3} \operatorname{Tr}\left(\mathcal{P}^{2}\right)
\end{aligned}
$$


and

$$
\mathcal{L}_{\text {brane }}=\sum_{i} e_{4} \delta\left(y-y_{i}\right)\left(-\lambda_{i}-\bar{\Psi}_{\mu}^{A} \gamma^{\mu \nu}\left(M_{i}+\gamma_{5} \bar{M}_{i}\right)_{A}^{B} \Psi_{\nu B}\right)
$$

The $M_{i}, \bar{M}_{i}$ are constant matrices, symmetric in the symplectic indices, that denote gravitini mass terms on the brane at the fixed point $y_{i}$. The covariant derivative contains both gravitational and gauge connections:

$$
D_{\alpha} \Psi_{\beta}^{A}=\nabla_{\alpha} \Psi_{\beta}^{A}+A_{\alpha} \mathcal{P}_{B}^{A} \Psi_{\beta}^{B},
$$

where $\mathcal{P}=\mathcal{P}_{a} \mathrm{i} \sigma^{a}$ is the gauge prepotential. The pair of gravitini satisfies symplectic Majorana condition $\bar{\Psi}^{A} \equiv \Psi_{A}^{\dagger} \gamma_{0}=\left(\epsilon^{A B} \Psi_{B}\right)^{T} C$ where $C$ is the charge conjugation matrix and $\epsilon^{A B}$ is the antisymmetric $S U(2)_{R}$ metric (we use the convention $\epsilon_{12}=\epsilon^{12}=1$ ). Supersymmetry transformations include singular terms proportional to the delta functions

$$
\begin{aligned}
\delta e_{\alpha}^{m} & =\frac{1}{2} \bar{\eta}^{A} \gamma^{m} \Psi_{\alpha A}, \quad \delta A_{\alpha}=-\frac{\mathrm{i}}{2 \sqrt{2}} \bar{\Psi}_{\alpha}^{A} \eta_{A}, \\
\delta \Psi_{\alpha}^{A} & =D_{\alpha} \eta^{A}-\frac{\mathrm{i}}{4 \sqrt{2}}\left(\gamma_{\alpha}^{\beta \gamma}-4 \delta_{\alpha}^{\beta} \gamma^{\gamma}\right) \mathcal{F}_{\beta \gamma} \eta^{A}+\frac{\sqrt{2} \mathrm{i}}{3} \mathcal{P}^{A B} \gamma_{\alpha} \eta_{B} \\
& +\epsilon^{-1}(y) \delta_{\alpha}^{5} \sum_{i} a_{i} \delta\left(y-y_{i}\right)\left(Q_{i}-\gamma_{5} \delta\right)_{B}^{A} \gamma_{5} \eta^{B},
\end{aligned}
$$

where $a_{i}=1$ if the orbifold step function $\epsilon(y)$ 'jumps up' at the fixed point $y_{i}$, or $a_{i}=-1$ if it 'jumps down'. The $Q_{i}$ denotes $\mathbf{Z}_{2}$ parity operator acting locally on the gravitini sector ${ }^{1}$ as follows:

$$
\Psi_{\mu}^{A}\left(y_{i}-y\right)=\gamma_{5}\left(Q_{i}\right)_{B}^{A} \Psi_{\mu}^{B}\left(y_{i}+y\right), \quad \Psi_{5}^{A}\left(y_{i}-y\right)=-\gamma_{5}\left(Q_{i}\right)_{B}^{A} \Psi_{5}^{B}\left(y_{i}+y\right) .
$$

The symplectic Majorana condition and the normalization $\left(Q_{i}\right)^{2}=1 \mathrm{imply} Q_{i}=\left(q_{i}\right)_{a} \sigma^{a}$, where $\left(q_{i}\right)_{a}$ are real parameters.

In the general case $\left[5\right.$ one can write down the prepotential as follows: $\mathcal{P}=g_{R} \epsilon(y) \mathcal{R}+g_{S} \mathcal{S}$, where $\mathcal{R}=r_{a} \mathrm{i} \sigma^{a}$ commutes and $\mathcal{S}=s_{a} \mathrm{i} \sigma^{a}$ anticommutes with each $Q_{i}$.

The closure of the supersymmetry algebra provides relations between parameters of the boundary Lagrangian and the prepotential:

$$
\begin{aligned}
& 0=\delta\left(y-y_{i}\right) \bar{\Psi}_{\mu}^{A} \gamma^{\mu}\left[\left(M_{i}-\gamma_{5} \bar{M}_{i}\right)_{A}^{B} \mathcal{P}_{B}^{C}+a_{i} \frac{1}{2} \epsilon^{-1}(y) \mathcal{P}_{A}^{B}\left(Q_{i}+\gamma_{5} \delta\right)_{B}^{C}+a_{i} g_{R} \gamma_{5} \mathcal{R}_{A}^{C}\right. \\
& +\frac{\mathrm{i}}{4 \sqrt{2}} \lambda_{i} \delta_{A}^{C}+a_{i} \frac{\mathrm{i}}{4 \sqrt{2}} \lambda_{i} \epsilon(y)\left(\gamma_{5}\left(M_{i}\right)_{A}^{C}-\left(\bar{M}_{i}\right)_{A}^{C}\right) \\
& \left.+\frac{\mathrm{i}}{4 \sqrt{2}} \lambda_{i}\left(\frac{1}{2} \gamma_{5}\left(Q_{i}\right)_{A}^{C}+\frac{1}{2} \delta_{A}^{C}\right)\right] \eta_{C}
\end{aligned}
$$

Let us assume the prepotential of the form $\mathcal{P}_{A}^{B}=g \mathrm{i}\left(\sigma_{1}\right)_{A}^{B}$ and $\left(Q_{0}\right)_{A}^{B}=\left(Q_{\pi}\right)_{A}^{B}=\left(\sigma_{3}\right)_{A}^{B}$. Let us allow only the even components of gravitini to have mass terms on the branes

$$
\left(M_{0, \pi}\right)_{A}^{B}=\frac{1}{2} \alpha_{0, \pi}\left(\sigma_{1}\right)_{A}^{B}, \quad\left(\bar{M}_{0, \pi}\right)_{A}^{B}=\frac{1}{2} \mathrm{i} \alpha_{0, \pi}\left(\sigma_{2}\right)_{A}^{B} .
$$

\footnotetext{
${ }^{1}$ The parameters $\eta^{A}$ of the supersymmetry transformations obey the same boundary conditions as the $4 \mathrm{~d}$ components of gravitini.
} 
Then the boundary conditions take the form

$$
\begin{aligned}
& \epsilon^{-1}(y) \delta(y) \gamma_{5}\left(\eta_{-}\right)^{A}=-\delta(y) \alpha_{0}\left(\sigma_{1}\right)_{B}^{A}\left(\eta_{+}\right)^{B}, \\
& \epsilon^{-1}(y) \delta\left(y-\pi r_{c}\right) \gamma_{5}\left(\eta_{-}\right)^{A}=\delta\left(y-\pi r_{c}\right) \alpha_{\pi}\left(\sigma_{1}\right)_{B}^{A}\left(\eta_{+}\right)^{B}
\end{aligned}
$$

where we have decomposed fermions into the even $(+)$ and odd $(-)$ components

$$
\left(\eta_{ \pm}\right)^{A}=\frac{1}{2}\left(\delta \pm \gamma_{5} \sigma_{3}\right)_{B}^{A} \eta^{B}
$$

The equations (77) are satisfied, if

$$
\lambda_{0}=-g 4 \sqrt{2} \frac{2 \alpha_{0}}{1+\alpha_{0}^{2}}, \quad \lambda_{\pi}=-g 4 \sqrt{2} \frac{2 \alpha_{\pi}}{1+\alpha_{\pi}^{2}} .
$$

For $g=\frac{3}{4} \sqrt{2} k$ the bosonic part of the Lagrangian reads

$$
S=\int d^{5} x \sqrt{-g_{5}}\left(\frac{1}{2} R+6 k^{2}\right)-6 \int d^{5} x \sqrt{-g_{4}} k\left(T_{0} \delta(y)+T_{\pi} \delta\left(y-\pi r_{c}\right)\right)
$$

where $T_{0, \pi}=-2 \alpha_{0, \pi} /\left(1+\alpha_{0, \pi}^{2}\right)$. Note that $\left|T_{0, \pi}\right| \leq 1$. For $T_{0}=-T_{\pi}=1$ we obtain supersymmetric Randall-Sundrum model, while for other values of $T_{0, \pi}$ we have $A d S_{5}$ in the bulk with $A d S_{4}$ foliations (the super-bigravity for example).

\section{Supersymmetry breakdown}

Let us assume a nonzero expectation value of $A_{5}$. Let us solve Killing equation to check whether supersymmetry remains unbroken:

$$
D_{\alpha} \eta^{A}-\frac{\sqrt{2} \mathrm{i}}{3} \mathcal{P}_{B}^{A} \gamma_{\alpha} \eta^{B}-\left(\delta(y)-\delta\left(y-\pi r_{c}\right)\right) \epsilon^{-1}(y) \delta_{\alpha}^{5}\left(\delta-\sigma_{3} \gamma_{5}\right)_{B}^{A} \eta^{B}=0
$$

For the RS background $\left(\alpha_{0}=-\alpha_{\pi}=-1\right)$ we can write

$$
\begin{aligned}
& 0=\partial_{\mu} \eta_{ \pm}^{A}-\frac{1}{2} k \epsilon(y) \gamma_{\mu} \gamma_{5} \eta_{\mp}^{A}+\frac{1}{2} k\left(\sigma_{1}\right)_{B}^{A} \gamma_{\mu} \eta_{ \pm}^{B} \\
& 0=\partial_{5} \eta_{+}^{A}+\mathrm{i} e\left(\sigma_{1}\right)_{B}^{A} \eta_{-}^{B}+\frac{1}{2} k\left(\sigma_{1}\right)_{B}^{A} \gamma_{5} \eta_{-}^{B} \\
& 0=\partial_{5} \eta_{-}^{A}+\mathrm{i} e\left(\sigma_{1}\right)_{B}^{A} \eta_{+}^{B}+\frac{1}{2} k\left(\sigma_{1}\right)_{B}^{A} \gamma_{5} \eta_{+}^{B}-2\left(\delta(y)-\delta\left(y-\pi r_{c}\right)\right) \epsilon^{-1}(y) \eta_{-}^{A}
\end{aligned}
$$

where $e=g\left\langle A_{5}\right\rangle$. The equation (14) is satisfied by $\eta_{-}^{A}=\epsilon(y) \gamma_{5}\left(\sigma_{1}\right)_{B}^{A} \eta_{+}^{B}$, where we have assumed that the Killing spinor doesn't depend on $x_{\mu}$. One can easily find the solution of (15) and (16)

$$
\begin{array}{ll}
\eta_{+}^{1}=e^{-\frac{1}{2}(k+2 i e)|y|} \hat{\eta}_{R}, & \eta_{-}^{1}=\epsilon(y) e^{-\frac{1}{2}(k-2 i e)|y|} \hat{\eta}_{L} \\
\eta_{+}^{2}=-e^{-\frac{1}{2}(k-2 i e)|y|} \hat{\eta}_{L}, & \eta_{-}^{2}=\epsilon(y) e^{-\frac{1}{2}(k+2 i e)|y|} \hat{\eta}_{R}
\end{array}
$$


where $\hat{\eta}$ is a four-dimensional Majorana spinor in flat space. Notice, that first and second components of the Killing spinors have phases, which are complex conjugates of each other. In fact, this relation is required by the $5 \mathrm{~d}$ Majorana condition. Thus there exists a global unbroken supersymmetry that gives rise to a flat $\mathrm{N}=1$ supergravity in $4 \mathrm{~d}$ with susy preserving vacua.

Let us now turn to the detuned case. As an example we consider the super-bigravity. Taking

$$
\alpha_{0}=-\frac{\cosh \left(k \pi r_{c} / 2\right) \pm 1}{\sinh \left(k \pi r_{c} / 2\right)}, \quad \alpha_{\pi}=-\frac{\cosh \left(k \pi r_{c} / 2\right) \pm 1}{\sinh \left(k \pi r_{c} / 2\right)}
$$

we obtain the bosonic action of the $4 \mathrm{~d}$ bigravity

$$
S=\int d^{5} x \sqrt{-g_{5}}\left(\frac{1}{2} R+6 k^{2}\right)-6 \int d^{5} x \sqrt{-g_{4}} k T\left(\delta(y)+\delta\left(y-\pi r_{c}\right)\right)
$$

where $k=\frac{2 \sqrt{2}}{3} g$ and $T=\tanh \left(k \pi r_{c} / 2\right)$. Gravitational background does not admit a flat $4 \mathrm{~d}$ Minkowski foliation, and the consistent solution is that of $A d S_{4}$ branes:

$$
d s^{2}=a^{2}(y) \bar{g}_{\mu \nu} d x^{\mu} d x^{\nu}+d y^{2}
$$

where

$$
a(y)=\frac{\sqrt{-\bar{\Lambda}}}{k} \cosh \left(k|y|-\frac{k \pi r_{c}}{2}\right),
$$

and $\bar{g}_{\mu \nu} d x^{\mu} d x^{\nu}=\exp \left(-2 \sqrt{-\bar{\Lambda}} x_{3}\right)\left(-d t^{2}+d x_{1}^{2}+d x_{2}^{2}\right)+d x_{3}^{2}$ is the four-dimensional AdS metric. The radius of the fifth dimension is determined in terms of the brane tensions

$$
k \pi r_{c}=\ln \left(\frac{1+T}{1-T}\right)
$$

Normalization $a(0)=1$ leads to the fine-tuning relation $\bar{\Lambda}=\left(T^{2}-1\right) k^{2}<0$.

Notice in (18), that we have two possibilities for the brane gravitini masses: $\alpha_{0}=1 / \alpha_{\pi}$ and $\alpha_{0}=\alpha_{\pi}$. In the first case five-dimensional vacuum spontaneously breaks all supersymmetries, while in the second $\mathrm{N}=1$ supersymmetry is preserved. To justify this observation, let us assume $\alpha_{0}=\alpha_{\pi}=-\alpha$, where

$$
\alpha=\frac{\cosh \left(k \pi r_{c} / 2\right)-1}{\sinh \left(k \pi r_{c} / 2\right)} .
$$

Killing equations in the presence of a non-zero expectation value of the $\left\langle A_{5}\right\rangle=e / g$, take the form

$$
\begin{aligned}
& 0=\bar{\nabla}_{\mu} \eta_{ \pm}^{A}+\frac{1}{2} k \epsilon(y) \tanh \left(k|y|-\frac{k \pi r_{c}}{2}\right) \gamma_{\mu} \gamma_{5} \eta_{\mp}^{A}+\frac{1}{2} k\left(\sigma_{1}\right)_{B}^{A} \gamma_{\mu} \eta_{ \pm}^{B} \\
& 0=\partial_{5} \eta_{+}^{A}+\mathrm{i} e\left(\sigma_{1}\right)_{B}^{A} \eta_{-}^{B}+\frac{1}{2} k\left(\sigma_{1}\right)_{B}^{A} \gamma_{5} \eta_{-}^{B} \\
& 0=\partial_{5} \eta_{-}^{A}+\mathrm{i} e\left(\sigma_{1}\right)_{B}^{A} \eta_{+}^{B}+\frac{1}{2} k\left(\sigma_{1}\right)_{B}^{A} \gamma_{5} \eta_{+}^{B}-2\left(\delta(y)-\delta\left(y-\pi r_{c}\right)\right) \epsilon^{-1}(y) \eta_{-}^{A}
\end{aligned}
$$


where $\bar{\nabla}_{\mu}$ denotes covariant derivative with respect to the four-dimensional AdS geometry. Note that the equations have imaginary coefficients, hence we have to consider complex amplitudes in the solution. The appropriate decomposition of the Killing spinors reads

$$
\eta_{+}^{A}=\left(\begin{array}{c}
\phi_{+}(y) \hat{\eta}_{R} \\
-\phi_{+}^{\star}(y) \hat{\eta}_{L}
\end{array}\right)^{A}, \quad \eta_{-}^{A}=\epsilon(y)\left(\begin{array}{c}
\phi_{-}^{\star}(y) \hat{\eta}_{L} \\
\phi_{-}(y) \hat{\eta}_{R}
\end{array}\right)^{A}
$$

where $\hat{\eta}$ denotes the Killing spinor in the $A d S_{4}$ which satisfies: $\left(\bar{\nabla}_{\mu}-\frac{1}{2} \sqrt{-\Lambda} \hat{\gamma}_{\mu}\right) \hat{\eta}=0$.

The equations (24), (25) and (26) can be solved by

$$
\begin{aligned}
& \phi_{+}(y)=N \cos (e|y|) \cosh \left(\frac{k|y|}{2}-\frac{k \pi r_{c}}{4}\right)+\mathrm{i} N \sin (e|y|) \sinh \left(\frac{k|y|}{2}-\frac{k \pi r_{c}}{4}\right), \\
& \phi_{-}(y)=-N \cos (e|y|) \sinh \left(\frac{k|y|}{2}-\frac{k \pi r_{c}}{4}\right)-\mathrm{i} N \sin (e|y|) \cosh \left(\frac{k|y|}{2}-\frac{k \pi r_{c}}{4}\right),
\end{aligned}
$$

where $N$ is a normalization constant. One can easily check that boundary conditions (9) are satisfied only if

$$
\frac{\sin \left(e \pi r_{c}\right)}{\cosh \left(k \pi r_{c} / 4\right)}=0
$$

which means that the $\left\langle A_{5}\right\rangle$ background does not break supersymmetry for $e=p / r_{c}$, where $p$ is an integer $(p \in \mathbf{Z})$, or in the limit $r_{c} \rightarrow \infty$, which implies $\lambda_{0 / \pi} \rightarrow 6 k$.

\section{Singular gauge transformations}

Typically, especially in models considered on $\mathbf{S}^{\mathbf{1}}$, one can break supersymmetry by a non-zero expectation value of $A_{5}$. To see why the situation is more complicated on an orbifold $\mathbf{S}^{\mathbf{1}} / \mathbf{Z}_{\mathbf{2}}$, let us attempt to go to the picture where $\left\langle A_{5}\right\rangle=0$. We can try to do this using gauge transformation of the form, [9],

$$
\Psi_{M} \longrightarrow e^{\mathcal{P} \Omega(y)} \Psi_{M}, \quad \eta \longrightarrow e^{\mathcal{P} \Omega(y)} \eta
$$

with $\mathcal{P}_{A}^{B}=g \mathrm{i}\left(\sigma_{1}\right)_{A}^{B}$, where we consider the most general form of the transformation parameter $\Omega(y)=\omega(|y|) \epsilon(y)$. Note, that in this paper by a gauge transformation we mean exclusively transformations which are 'legal' on the orbifold, i.e. the ones which are periodic and preserve $Z_{2}$-parities of all fields. We will find the explicit form of the $\Omega(y)$ requiring that $\delta A_{5}=-e / g$ and gauge invariance of the bulk plus brane action.

To be consistent with the 'epsilon rules' (see [10]), we have to require that gauge transformations do not change the powers of the $\epsilon(y)$

$$
\begin{aligned}
& \Psi_{A}^{+} \longrightarrow \Psi_{A}^{+}+\mathrm{i} g \omega \epsilon^{-1}\left(\sigma_{1}\right)_{A}^{B} \Psi_{B}^{-}, \\
& \Psi_{A}^{-} \longrightarrow \Psi_{A}^{-}+\mathrm{i} g \omega \epsilon\left(\sigma_{1}\right)_{A}^{B} \Psi_{B}^{+} .
\end{aligned}
$$

One can write the finite transformations as follows

$$
\begin{aligned}
\Psi_{A}^{ \pm} \longrightarrow \quad & \frac{1}{2}\left(\delta \pm \gamma_{5} \sigma_{3}\right) e^{\mathrm{i} g \sigma_{1} \omega \epsilon} \Psi_{A}=\sum_{n=0}^{\infty} \frac{(\mathrm{i} g \omega)^{2 n}}{(2 n) !} \Psi_{A}^{ \pm}+\sum_{n=0}^{\infty} \frac{(\mathrm{i} g \omega)^{2 n+1}}{(2 n+1) !} \epsilon^{\mp 1} \sigma_{1} \Psi_{A}^{\mp} \\
& =\cos (g \omega) \Psi_{A}^{ \pm}+\mathrm{i} \epsilon^{\mp 1} \sin (g \omega) \sigma_{1} \Psi_{A}^{\mp} .
\end{aligned}
$$


Notice, that in this case we should treat $e^{\mathrm{i} g \sigma_{1} \omega \epsilon}$ not as a function of $\epsilon$, but rather as a symbolic shorthand for the expression (32). If we restrict ourselves to the bulk Lagrangian, both forms coincide. The difference becomes important for the singular terms proportional to the $\delta(y)$ function.

The Lagrangian variation under gauge transformations includes:

$$
\begin{aligned}
& \frac{1}{2}\left(\bar{\Psi}_{ \pm}\right)_{\mu}^{A} \gamma^{\mu \nu} \gamma^{5} \partial_{5}\left(\Psi_{\mp}\right)_{\nu A}+\frac{1}{2}\left(\bar{\Psi}_{ \pm}\right)_{\mu}^{A} \gamma^{\mu \nu} \gamma^{5} A_{5} \mathrm{i} g\left(\sigma_{1}\right)_{A}^{B}\left(\Psi_{ \pm}\right)_{\nu B} \\
& -\left(\bar{\Psi}_{+}\right)_{\mu}^{A} \gamma^{\mu \nu}\left(\sigma_{1}\right)_{A}^{B}\left(\Psi_{+}\right)_{\nu B}\left(\alpha_{0} \delta(y)+\alpha_{\pi} \delta\left(y-\pi r_{c}\right)\right) \longrightarrow \\
& \longrightarrow \frac{1}{2}\left(\bar{\Psi}_{ \pm}\right)_{\mu}^{A} \gamma^{\mu \nu} \gamma^{5} \partial_{5}\left(\Psi_{\mp}\right)_{\nu A}+\frac{1}{2}\left(\bar{\Psi}_{ \pm}\right)_{\mu}^{A} \gamma^{\mu \nu} \gamma^{5} \mathrm{i} g \omega^{\prime} \epsilon\left(\sigma_{1}\right)_{A}^{B}\left(\Psi_{ \pm}\right)_{\nu B} \\
& \pm \mathrm{i} \cos (g \omega) \sin (g \omega)\left(\bar{\Psi}_{ \pm}\right)_{\mu}^{A} \gamma^{\mu \nu} \gamma^{5} \sigma_{1}\left(\Psi_{ \pm}\right)_{\nu A} \epsilon^{-1} \epsilon^{ \pm 1}\left(\delta(y)-\delta\left(y-\pi r_{c}\right)\right) \\
& \pm \sin ^{2}(g \omega)\left(\bar{\Psi}_{\mp}\right)_{\mu}^{A} \gamma^{\mu \nu} \gamma^{5}\left(\Psi_{ \pm}\right)_{\nu A} \epsilon^{-1}\left(\delta(y)-\delta\left(y-\pi r_{c}\right)\right) \\
& +\frac{1}{2}\left(\bar{\Psi}_{ \pm}\right)_{\mu}^{A} \gamma^{\mu \nu} \gamma^{5} A_{5} \mathrm{i} g\left(\sigma_{1}\right)_{A}^{B}\left(\Psi_{ \pm}\right)_{\nu B}-\frac{1}{2}\left(\bar{\Psi}_{ \pm}\right)_{\mu}^{A} \gamma^{\mu \nu} \gamma^{5} \mathrm{i} e\left(\sigma_{1}\right)_{A}^{B}\left(\Psi_{ \pm}\right)_{\nu B} \\
& -\cos ^{2}(g \omega)\left(\bar{\Psi}_{+}\right)_{\mu}^{A} \gamma^{\mu \nu}\left(\sigma_{1}\right)_{A}^{B}\left(\Psi_{+}\right)_{\nu B}\left(\alpha_{0} \delta(y)+\alpha_{\pi} \delta\left(y-\pi r_{c}\right)\right) \\
& -\sin ^{2}(g \omega)\left(\bar{\Psi}_{-}\right)_{\mu}^{A} \gamma^{\mu \nu}\left(\sigma_{1}\right)_{A}^{B}\left(\Psi_{-}\right)_{\nu B} \epsilon^{-2}\left(\alpha_{0} \delta(y)+\alpha_{\pi} \delta\left(y-\pi r_{c}\right)\right) \\
& \mp \mathrm{i} \cos (g \omega) \sin (g \omega)\left(\bar{\Psi}_{ \pm}\right)_{\mu}^{A} \gamma^{\mu \nu}\left(\Psi_{\mp}\right)_{\nu A} \epsilon^{-1}\left(\alpha_{0} \delta(y)+\alpha_{\pi} \delta\left(y-\pi r_{c}\right)\right) \text {. }
\end{aligned}
$$

Gauge invariance in the bulk requires

$$
\omega^{\prime}(y)=\frac{e}{g} \epsilon(y) \Longrightarrow \omega(y)=\frac{e}{g}|y| .
$$

One can verify, that such a transformation removes imaginary phases in the solution (177).

It is worth noticing that in terms of the odd parameter $\Omega$, the gauge transformation of the $A_{5}$ reads

$$
A_{5} \longrightarrow A_{5}-\epsilon \partial_{5}\left(\Omega \epsilon^{-1}\right)
$$

One can check that on the branes the Lagrangian is not gauge invariant for $\left|\alpha_{0, \pi}\right| \neq 1$, and the uncancelled variation reads

$$
\begin{aligned}
\delta \mathcal{L}=\quad & \mathrm{i} \cos (g \omega) \sin (g \omega)\left(\bar{\Psi}_{+}\right)_{\mu}^{A} \gamma^{\mu \nu} \gamma^{5} \sigma_{1}\left(\Psi_{+}\right)_{\nu A}\left(\left(1-\alpha_{0}^{2}\right) \delta(y)-\left(1-\alpha_{\pi}^{2}\right) \delta\left(y-\pi r_{c}\right)\right) \\
& +\sin ^{2}(g \omega)\left(\bar{\Psi}_{-}\right)_{\mu}^{A} \gamma^{\mu \nu} \gamma^{5}\left(\Psi_{+}\right)_{\nu A} \epsilon^{-1}\left(\left(1-\alpha_{0}^{2}\right) \delta(y)-\left(1-\alpha_{\pi}^{2}\right) \delta\left(y-\pi r_{c}\right)\right)
\end{aligned}
$$

The above variation vanishes for $\sin (g \omega(0))=0$ and $\sin \left(g \omega\left(\pi r_{c}\right)\right)=0$. The same conclusion can be obtained in a different way. One can analyze the action of gauge transformations on the boundary conditions (9). These change under the gauge transformation

$$
\eta_{A}^{ \pm} \longrightarrow \cos (g \omega) \eta_{A}^{ \pm}+\mathrm{i} \epsilon^{\mp 1} \sin (g \omega) \sigma_{1} \eta_{A}^{\mp}
$$

into

$$
\begin{aligned}
& \epsilon^{-1} \delta(y) \cos (g \omega) \gamma_{5}\left(\eta^{-}\right)^{A}+\mathrm{i} \delta(y) \sin (g \omega)\left(\sigma_{1}\right)_{B}^{A} \gamma_{5}\left(\eta^{+}\right)^{B} \\
& \quad=-\delta(y) \alpha_{0} \cos (g \omega)\left(\sigma_{1}\right)_{B}^{A}\left(\eta^{+}\right)^{B}-\mathrm{i} \epsilon^{-1} \delta(y) \alpha_{0} \sin (g \omega)\left(\eta^{-}\right)^{A} \\
& \epsilon^{-1} \delta(y- \\
& \left.\quad \pi r_{c}\right) \cos (g \omega) \gamma_{5}\left(\eta^{-}\right)^{A}+\mathrm{i} \delta\left(y-\pi r_{c}\right) \sin (g \omega)\left(\sigma_{1}\right)_{B}^{A} \gamma_{5}\left(\eta^{+}\right)^{B} \\
& \quad=\delta\left(y-\pi r_{c}\right) \alpha_{\pi} \cos (g \omega)\left(\sigma_{1}\right)_{B}^{A}\left(\eta^{+}\right)^{B}+\mathrm{i} \epsilon^{-1} \delta\left(y-\pi r_{c}\right) \alpha_{\pi} \sin (g \omega)\left(\eta^{-}\right)^{A} .
\end{aligned}
$$


These boundary conditions are invariant under the gauge transformation if $\sin (g \omega(0))=0$ and $\sin \left(g \omega\left(\pi r_{c}\right)\right)=0$, or for $\left|\alpha_{0, \pi}\right|=1$. For the specific $\omega(y)$ given by (34) we obtain quantization condition for the allowed backgrounds $e_{p}$, which singles out gauge transformations which do not change boundary conditions:

$$
e_{p}=p / r_{c}
$$

where $p \in \mathbf{Z}$. We can parametrize different classes of the detuned models by a parameter $\theta \in\left\langle 0, \frac{1}{g r_{c}}\right)$ such that for given $\alpha_{0, \pi}$ the vacuum expectation value of $A_{5}$ equals $\left\langle A_{5}\right\rangle=\theta+\frac{p}{g r_{c}}$, where $p \in \mathbf{Z}$. Models belonging to the same class are connected to each other by transformations (37) with (34) and (39). For $\alpha_{0}=\alpha_{\pi}=-\alpha$ the class with unbroken $N=1$ supersymmetry is labelled by $\theta=0$. One can check that $\theta=\frac{1}{2 g r_{c}}$ corresponds to the flipped super-bigravity. The redefinition (an 'illegal' gauge transformation)

$$
\begin{aligned}
& \eta_{A}^{ \pm} \longrightarrow \cos \left(\frac{|y|}{2 r_{c}}\right) \eta_{A}^{ \pm}+\mathrm{i} \epsilon^{\mp 1} \sin \left(\frac{|y|}{2 r_{c}}\right) \sigma_{1} \eta_{A}^{\mp}, \\
& A_{5} \longrightarrow A_{5}-\frac{1}{2 g r_{c}}
\end{aligned}
$$

transforms this model to the frame, where $\alpha_{0}=1 / \alpha_{\pi}=-\alpha$ with $\left\langle A_{5}\right\rangle=0$. In the same way one can show that configuration $\alpha_{0}=1 / \alpha_{\pi}=-\alpha$ with $\left\langle A_{5}\right\rangle=\frac{1}{2 g r_{c}}$ corresponds, upon the same redefinition, to the configuration $\alpha_{0}=\alpha_{\pi}=-\alpha$ with vanishing $\left\langle A_{5}\right\rangle$, where $\mathrm{N}=1$ supersymmetry is unbroken.

It is worth noticing that even for a function $\omega$ which is independent of the fifth coordinate, the brane Lagrangian is not invariant under the gauge transformation as long as $\left|\alpha_{0}\right| \neq 1$ or $\left|\alpha_{\pi}\right| \neq 1$, in fact even the global $U(1)$ symmetry is explicitly broken. Supersymmetry breakdown by a non-zero vacuum expectation value of $A_{5}$ can be understood as a consequence of the explicit breaking of the gauged $U(1)$ symmetry. To be more explicit, let us assume that for some values of the parameters $\alpha_{0}, \alpha_{\pi}$ and $\left\langle A_{5}\right\rangle=e / g$, the $\mathrm{N}=1$ supersymmetry stays unbroken, and that there exists a Killing spinor which satisfies boundary conditions generated by $\alpha_{0}$ and $\alpha_{\pi}$. Let us imagine choosing another expectation value of $A_{5}\left(\left\langle A_{5}\right\rangle=e^{\prime} / g\right)$ such that $e^{\prime}-e \neq \frac{p}{r_{c}}$, where $p \in \mathbf{Z}$. One can gauge-transform this model to the frame where $\left\langle A_{5}\right\rangle=e / g$. The bulk Lagrangian is invariant under such a transformation, hence the solution for the Killing spinor in the bulk remains unchanged. However, the brane Lagrangian is not invariant and one obtains different boundary conditions with $\alpha_{0}^{\prime} \neq \alpha_{0}$ or $\alpha_{\pi}^{\prime} \neq \alpha_{\pi}$ ('prime' denotes parameters after the transformation), which are not satisfied by the bulk solution and, consequently, supersymmetry must be broken. On the other hand, in the 'tuned' case one can always gauge away the nonzero vacuum expectation value of $A_{5}$, hence, if supersymmetry is unbroken for some $\left\langle A_{5}\right\rangle$, this implies that it remains unbroken for any $\left\langle A_{5}\right\rangle$.

\section{Supersymmetric Randall-Sundrum model with $\mathrm{Z}_{2}$-odd prepotential}

It is interesting to check whether the same analysis can be repeated for the FLP model. Let us take the prepotential $\mathcal{P}_{A}^{B}=g \mathrm{i} \epsilon(y)\left(\sigma_{3}\right)_{A}^{B}$ and $\left(Q_{0}\right)_{A}^{B}=\left(Q_{\pi}\right)_{A}^{B}=\left(\sigma_{3}\right)_{A}^{B}$. We do not put any 
gravitini mass terms on the brane. The equations (17) reduce to

$$
\lambda_{0}=g 4 \sqrt{2}, \quad \lambda_{\pi}=-g 4 \sqrt{2} .
$$

For $g=\frac{3}{4} \sqrt{2} k$ we obtain the bosonic part of the Randall-Sundrum model.

Let us assume nonzero expectation value of $A_{5}=e / g$. Killing equation in the RS background reads

$$
\begin{aligned}
& 0=\partial_{\mu} \eta_{ \pm}^{A}-\frac{1}{2} k \epsilon(y) \gamma_{\mu} \gamma_{5} \eta_{\mp}^{A}+\frac{1}{2} k \epsilon(y)\left(\sigma_{3}\right)_{B}^{A} \gamma_{\mu} \eta_{\mp}^{B} \\
& 0=\partial_{5} \eta_{ \pm}^{A}+\operatorname{ie} \epsilon(y)\left(\sigma_{3}\right)_{B}^{A} \eta_{ \pm}^{B}+\frac{1}{2} k \epsilon(y)\left(\sigma_{3}\right)_{B}^{A} \gamma_{5} \eta_{ \pm}^{B}
\end{aligned}
$$

The equation (43) implies $\eta_{-}^{A}=0$, where it has been assumed that the Killing spinor doesn't depend on $x_{\mu}$. One can easily find the solution for $\eta_{+}^{A}$

$$
\eta_{+}^{1}=e^{-\frac{1}{2}(k+2 i e)|y|} \hat{\eta}_{R}, \quad \eta_{+}^{2}=-e^{-\frac{1}{2}(k-2 i e)|y|} \hat{\eta}_{L}
$$

where $\hat{\eta}$ is a four-dimensional Majorana spinor in flat space.

Let us turn to the picture where $\left\langle A_{5}\right\rangle=0$. We can do this using the gauge transformation

$$
\Psi_{M} \longrightarrow e^{\mathcal{P} \Omega(y)} \Psi_{M}, \quad \eta \longrightarrow e^{\mathcal{P} \Omega(y)} \eta
$$

In this case we do not face problems with the singular terms, because we do not consider gravitini mass terms on the brane and $\partial_{5}\left(e^{\mathcal{P} \Omega(y)}\right)$ does not produce $\delta(y)$ function $(\mathcal{P} \Omega(y)$ is even). So, we can simply write finite transformation as follows:

$$
\Psi_{A}^{ \pm} \longrightarrow e^{\mathrm{i} \epsilon g \sigma_{3} \Omega} \Psi_{A}^{ \pm}, \quad A_{5} \longrightarrow A_{5}-\frac{e}{g}
$$

The Lagrangian variation under the $U(1)$ gauge transformation includes:

$$
\begin{aligned}
& \frac{1}{2}\left(\bar{\Psi}_{ \pm}\right)_{\mu}^{A} \gamma^{\mu \nu} \gamma^{5} \partial_{5}\left(\Psi_{\mp}\right)_{\nu A}+\frac{1}{2}\left(\bar{\Psi}_{ \pm}\right)_{\mu}^{A} \gamma^{\mu \nu} \gamma^{5} A_{5} \mathrm{i} \epsilon g\left(\sigma_{3}\right)_{A}^{B}\left(\Psi_{\mp}\right)_{\nu B} \longrightarrow \\
& \longrightarrow \frac{1}{2}\left(\bar{\Psi}_{ \pm}\right)_{\mu}^{A} \gamma^{\mu \nu} \gamma^{5} \partial_{5}\left(\Psi_{\mp}\right)_{\nu A}+\frac{1}{2}\left(\bar{\Psi}_{ \pm}\right)_{\mu}^{A} \gamma^{\mu \nu} \gamma^{5} \mathrm{i} g(\epsilon \Omega)^{\prime}\left(\sigma_{3}\right)_{A}^{B}\left(\Psi_{\mp}\right)_{\nu B} \\
& \quad+\frac{1}{2}\left(\bar{\Psi}_{ \pm}\right)_{\mu}^{A} \gamma^{\mu \nu} \gamma^{5} A_{5} \mathrm{i} \epsilon g\left(\sigma_{3}\right)_{A}^{B}\left(\Psi_{\mp}\right)_{\nu B}-\frac{1}{2}\left(\bar{\Psi}_{ \pm}\right)_{\mu}^{A} \gamma^{\mu \nu} \gamma^{5} \mathrm{i} \epsilon e\left(\sigma_{3}\right)_{A}^{B}\left(\Psi_{\mp}\right)_{\nu B}
\end{aligned}
$$

and gauge invariance requires

$$
(\epsilon(y) \Omega(y))^{\prime}=\frac{e}{g} \epsilon(y) \Longrightarrow \Omega(y)=\frac{e}{g} \epsilon(y)|y|
$$

hence, one can gauge away non-zero vacuum expectation value by a true gauge transformation.

One can check, [12, that turning on non-zero gravitini masses on the branes while retaining the $\mathbf{Z}_{\mathbf{2}}$-odd prepotential, explicitly violates the $U(1)$ (gauged and global) symmetry and, therefore, in such a case it becomes possible to break supersymmetry by the expectation value of $A_{5}$. 


\section{Summary}

In this note we have analyzed supersymmetry breakdown by nonzero vacuum expectation value of $A_{5}$ in five-dimensional warped supergravities on the orbifold $\mathbf{S}^{\mathbf{1}} / \mathbf{Z}_{\mathbf{2}}$. We have shown that typically a wide class of gauge transformations does not respect the boundary conditions (9), and, equivalently, the brane actions are not invariant under such transformations. As a consequence, it is not always possible to gauge away a nonzero vacuum expectation value of $A_{5}$. Under such circumstances supersymmetry is spontaneously broken and we can parametrize different classes of models by the parameter $\theta \in\left\langle 0, \frac{1}{g r_{c}}\right)$, such that for given gravitini masses on the branes the vacuum expectation value of $A_{5}$ is $\left\langle A_{5}\right\rangle=\theta+\frac{p}{g r_{c}}$ for $p \in \mathbf{Z}$. In the special 'tuned' case where $\left|\lambda_{0}\right|=\left|\lambda_{\pi}\right|=6 k$ all gauge transformations respect boundary conditions and supersymmetry remains unbroken for any value of $\left\langle A_{5}\right\rangle$. One may say, that the explicit breaking of the $U(1)$ gauge invariance is necessary for supersymmetry breakdown by $\left\langle A_{5}\right\rangle$. It is interesting to notice that the origin of supersymmetry violation can be traced back to fermionic boundary conditions given in terms of boundary mass parameters of gravitini, which are supersymmetry singlets, whereas boundary superpotentials known to play a similar role do transform under supersymmetry variations.

When this work was at the final stage of preparation, the very interesting paper [12] appeared, where the issue of supersymmetry breakdown by Wilson lines on warped $S^{1} / Z_{2}$ has been discussed.

\section{Acknowledgments}

Authors thank M. Quiros and F. Zwirner for interesting conversations. Z.L. thanks Theory Division at CERN for hospitality. This work was partially supported by the EC Contract HPRN-CT-2000-00152 for years 2000-2004, by the Polish State Committee for Scientific Research grants KBN 2P03B 00125 (Z.L.) and by KBN 2P03B 12425 (R.M.), and by POLONIUM 2003.

\section{References}

[1] R. Altendorfer, J. Bagger and D. Nemeschansky, "Supersymmetric Randall-Sundrum scenario," Phys. Rev. D 63 (2001) 125025 arXiv:hep-th/0003117.

[2] A. Falkowski, Z. Lalak and S. Pokorski, "Supersymmetrizing branes with bulk in fivedimensional supergravity," Phys. Lett. B 491 (2000) 172 |arXiv:hep-th/0004093|.

[3] E. Bergshoeff, R. Kallosh and A. Van Proeyen, "Supersymmetry in singular spaces," JHEP 0010 (2000) 033 |arXiv:hep-th/0007044.

[4] A. Lukas, B. A. Ovrut, K. S. Stelle and D. Waldram, "The universe as a domain wall," Phys. Rev. D 59 (1999) 086001 arXiv:hep-th/9803235. 
[5] P. Brax, A. Falkowski and Z. Lalak, "Non-BPS branes of supersymmetric brane worlds," Phys. Lett. B 521 (2001) 105 arXiv:hep-th/0107257.

[6] J. A. Bagger, F. Feruglio and F. Zwirner, "Generalized symmetry breaking on orbifolds," Phys. Rev. Lett. 88 (2002) 101601 arXiv:hep-th/0107128.

[7] K. A. Meissner, H. P. Nilles and M. Olechowski, "Brane induced supersymmetry breakdown and restoration," Acta Phys. Polon. B 33 (2002) 2435 arXiv:hep-th/0205166.

[8] A. Delgado, G. von Gersdorff and M. Quiros, "Brane-assisted Scherk-Schwarz supersymmetry breaking in orbifolds," JHEP 0212 (2002) 002 arXiv:hep-th/0210181.

[9] Z. Lalak and R. Matyszkiewicz, "On Scherk-Schwarz mechanism in gauged fivedimensional supergravity and on its relation to bigravity," Nucl. Phys. B 649 (2003) 389 arXiv:hep-th/0210053.

[10] Z. Lalak and R. Matyszkiewicz, "Twisted supergravity and untwisted super-bigravity," Phys. Lett. B 562 (2003) 347 arXiv:hep-th/0303227.

[11] J. Bagger and D. Belyaev, "Twisting warped supergravity," JHEP 0306 (2003) 013 arXiv:hep-th/0306063.

[12] J. Bagger and M. Redi, "Supersymmetry breaking by Wilson lines in AdS(5)," arXiv:hep-th/0310086.

[13] G. v. Gersdorff, M. Quiros and A. Riotto, "Scherk-Schwarz supersymmetry breaking with radion stabilization," arXiv:hep-th/0310190. 